\title{
Fatores associados à subnotificação de pacientes com Aids, no Rio de Janeiro, RJ, 1996
}

\section{Variables associated with underreporting of AIDS patients, Brazil, 1996}

\section{Vanja MB Ferreiraa, Margareth C Portela ${ }^{b}$ e Mauricio TL Vasconcellos}

aSecretaria de Estado de Saúde do Rio de Janeiro. Rio de Janeiro, RJ, Brasil. 'b epartamento de Administração e Planejamento em Saúde da Escola Nacional de Saúde Pública da Fundação O swaldo Cruz. Rio de Janeiro, RJ, Brasil. 'D epartamento de M etodologia da Fundação Instituto Brasileiro de Geografia e Estatística. Rio de Janeiro, RJ, Brasil 


\section{Fatores associados à subnotificação de pacientes com Aids, no Rio de Janeiro, RJ, 1996*}

\section{Variables associated with underreporting of AIDS patients, Brazil, 1996}

\section{Vanja MB Ferreiraa, Margareth C Portela ${ }^{b}$ e Mauricio TL Vasconcellos ${ }^{c}$}

aSecretaria de Estado de Saúde do Rio de Janeiro. Rio de Janeiro, RJ, Brasil. 'D epartamento de Administração e Planejamento em Saúde da Escola Nacional de Saúde Pública da Fundação O swaldo Cruz. Rio de Janeiro, RJ, Brasil. 'Departamento de Metodologia da Fundação Instituto Brasileiro de Geografia e Estatística. Rio de Janeiro, RJ, Brasil

\section{D escritores}

Síndrome de imunodeficiência adquirida, epidemiologia ${ }^{\#}$.

Notificação de doenças, estatísticas e dados numéricos ${ }^{\#}$. Sistemas de informação hospitalar".

Sistemas de informação em saúde. Pacientes internados. SUS (Br). Subnotificação.

\section{Resumo}

\section{Objetivo}

Existe uma expressiva subnotificação de casos de Aids no Município do Rio de Janeiro, RJ. Nesse sentido, foi realizado estudo com o objetivo de analisar os fatores associados a esse evento.

\section{Métodos}

Com base em dados do Sistema de Informações Hospitalares do Sistema Único de Saúde, referentes ao ano de 1996 e ao Município do Rio de Janeiro, e do Sistema de Informações sobre Agravos de Notificação, atualizado até setembro de 1997, selecionou-se uma amostra aleatória de prontuários, pela qual os pacientes foram classificados em notificados, não notificados e sem evidência para notificação. Foi utilizado um modelo multinomial para a análise das chances de ocorrência de subnotificação versus notificação e não-notificação sem evidência de diagnóstico de Aids versus notificação. Resultados

Não foram encontradas associações estatisticamente significativas entre as variáveis "idade", "estado civil", "escolaridade", "ocupação" e "gravidade da doença" e subnotificação de casos de Aids. A variável "sexo feminino" apresentou forte associação com as internações por procedimento Aids sem evidências para 'fechar caso' dessa doença. Foi encontrada associação bastante expressiva entre ter sido internado no mesmo hospital mais de uma vez e estar notificado. A presença de um setor de vigilância epidemiológica no hospital esteve inversamente associada à subnotificação de casos de Aids.

\section{Conclusões}

Os resultados mostraram que a associação significativa entre variáveis organizacionais e estruturais e subnotificação de casos de AIDS apontam para a necessidade de normatização de processos e fluxos, a fim de melhorar a qualidade do sistema de informações em saúde.

\footnotetext{
Abstract

Objective

The underreporting of AIDS cases in the municipality of Rio de Janeiro, Brazil, is significant. The study intends to analyze the factors associated to this event.
}

*Trabalho realizado no Departamento de Administração e Planejamento em Saúde da Escola N acional de Saúde Pública, Fundação O swaldo Cruz. Extraído da dissertação de mestrado, apresentada à Escola N acional de Saúde Pública, em 1999 Recebido em 27/1/1999. Reapresentado em 20/10/1999. A provado em 3/11/1999. 


\section{Keywords}

Acquired Immunodeficiency

Syndrome, epidemiology". Disease

notification, statistics \&

numerical data. Hospital

information systems ${ }^{\#}$.

\begin{abstract}
Methods
Using data provided by the Hospital Information System for the year of 1996, in Rio de Janeiro city, and by the National Surveillance System, patients were randomly selected and their medical records reviewed to verify an AIDS diagnosis. A multinomial model was used to perform an analysis of the variations on the chances of underreporting of AIDS cases versus reporting and on the chances of underreporting without evidences to fulfill the case definition of AIDS versus reporting.

Results

No significant associations were found between the variables such as "age", "marital status", "level of education", "occupation", and "severity of illness" and the underreporting of AIDS cases. The variable "female gender" showed a strong association with hospitalization without evidence of an AIDS diagnosis. A strong association was found between two or more admissions in an inpatient unit care and reporting. The existence of a epidemiological surveillance department in the hospital is inversely associated with the underreporting of AIDS cases.

Conclusion

The significant association between organizational variables and underreporting of AIDS cases found in the study point out to the need of standardization of the surveillance procedures, the especial need for the creation and maintenance of surveillance departments in hospitals to improve the quality of the health information system and, therefore, AIDS prevention and care.
\end{abstract}

\section{INTRO DU ÇÃO}

Em 1996, 21 unidades hospitalares, localizadas no Município do Rio de Janeiro, emitiram 3.789 autorizações de internação hospitalar (AIH) para tratamento de Aids, que corresponderam a 1.929 pacientes.

Com o objetivo de estimar a subnotificação de casos de Aids entre indivíduos hospitalizados pelo Sistema Único de Saúde (SUS), no Município do Rio de Janeiro, Ferreira \& Portela ${ }^{8}$ compararam as informações fornecidas pelo banco de dados do Sistema de Informações Hospitalares do SUS (SIH-SUS) em 1996, com os dados incluídos no Sistema de Informação de Agravos de Notificação (Sinan) da Secretaria Municipal de Saúde do Rio de Janeiro, até setembro de 1997. Os resultados do estudo apontaram para uma subnotificação de $42,8 \%$ de casos de Aids. Por meio de análises bivariadas, os citados autores identificaram associações entre a ocorrência de subnotificação e algumas variáveis administrativas (natureza jurídica do hospital), demográficas (sexo, idade, município de residência) e clínicas (diagnósticos principal e secundário, resultado da internação; variável "cobrança"). Dentre as associações estatisticamente significantes, chamou a atenção a ocorrência de um maior índice de subnotificação de casos de Aids em pacientes atendidos pelas unidades municipais.

Ainda que a Aids seja uma doença de notificação compulsória, no Brasil, desde 1984, o início da epidemia foi caracterizado por uma subnotificação expressiva de- vida ao preconceito, ao estigma da doença e ao nível socioeconômico da população primariamente atingida, que buscava atendimento nos consultórios particulares e nos planos de saúde. ${ }^{4}$ A partir da sucessiva elevação dos custos do tratamento e da pauperização da clientela atingida, ${ }^{7,13}$ aliado ao trabalho contínuo de desmistificação da doença e aos esforços governamentais para prover terapêutica específica gratuita aos pacientes, os índices de subnotificação diminuíram. ${ }^{9}$

No entanto, a discrepância entre o número de casos de Aids diagnosticados e o número de casos notificados, por atraso no registro ou subnotificação, é reconhecida por autores como Barbosa \& Struchiner, ${ }^{3}$ que analisaram métodos que corrigem o atraso da notificação.

Ao contrário do Sinan, o SIH-SUS não é universal, já que cobre apenas aquelas internações realizadas pela rede de serviços, financiada com recursos estatais. No entanto, essa cobertura é bastante extensa, estando calculada entre $70 \%$ e $80 \%,{ }^{10}$ índice que deve crescer, no caso da Aids, devido a já citada pauperização da epidemia. O achado de $42,8 \%$ de subnotificação na população atendida pelo SUS parece extremamente significativo e preocupante. Por outro lado, dada a agilidade do SIH-SUS, cujos dados podem chegar às mãos dos gestores de um a dois meses após sua emissão, a utilização das informações sobre as internações hospitalares se constitui em instrumento auxiliar valioso para a captação precoce de casos de Aids. 
O presente trabalho tem por objetivo estudar os fatores associados à subnotificação no Sinan de pacientes com internações por Aids, cobertas pelo SUS, no Município do Rio de Janeiro. Entretanto, assumese que o status de notificação não corresponde essencialmente a uma variável dicotômica, visto a possibilidade de pacientes internados para "tratamento da Aids" não possuírem, de fato, um diagnóstico da doença. ${ }^{11,12}$ Nesse sentido, o presente trabalho parte da reclassificação dos casos anteriormente identificados como subnotificados e estabelece, a partir de informações colhidas nos prontuários da população sob estudo, três status de notificação: (1) não notificados, (2) não notificados e sem evidências para definição de caso e (3) notificados.

\section{MÉTODOS}

\section{Dados}

A partir do banco de dados contendo as informações provenientes do SIH-SUS, e o status de notificação segundo o Sinan, foi selecionada uma amostra aleatória da população de 1.929 pacientes com hospitalizações por Aids cobertas pelo SUS, no Município do Rio de Janeiro, em 1996.

\section{Desenho da amostra}

Os pacientes que estiveram internados em mais de um hospital no período $(4,5 \%)$ foram relacionados ao local onde apresentaram o maior tempo de permanência, somadas todas as internações no ano. Das 21 unidades hospitalares identificadas, três foram excluídas devido ao inexpressivo número de $\mathrm{AIH}$ emitidas (menos de 10), reduzindo para 1.921 pacientes a população amostrada, segundo conceito definido por Cochran. ${ }^{6}$ Essa população foi estratificada por hospital e status de notificação, resultando em 36 estratos.

O desenho de amostra utilizado foi o de amostragem aleatória estratificada, com alocação proporcional ao número de pacientes de cada estrato na população. Esse desenho foi escolhido por assegurar as mesmas proporções da população amostrada e por gerar fatores de expansão constantes, que podem ser ignorados nos processos de análise que não visam a fazer inferências sobre o total populacional.

Para determinação do tamanho da amostra foi utilizada a expressão da variância do estimador, da proporção de casos não notificados, com erro relativo de $10 \%$ e nível de confiança de $95 \%$. Essa proporção variou, por hospital, entre 0,25 e 0,80 , sendo igual a 0,4211 para a população amostrada. O procedimento conduziu a uma amostra de 384 pacientes que, arre- dondada durante a alocação nos 36 estratos, resultou em 390 pacientes que representaram $680 \mathrm{AIH}$. Os pacientes foram selecionados com eqüiprobabilidade e sem reposição.

Realizado o trabalho de campo, observou-se perda de 13 prontuários em 8 dos 18 hospitais, sendo 7 de pacientes notificados e 6 de não notificados. Esse pequeno número de inexistência de respostas e de nãoconcentração em algum estrato não acarretou tendência na amostra, e resultou em aumento inexpressivo do erro de amostragem (de 10\% para 10,101\%).

\section{Revisão de prontuários}

A partir da variável "número do prontuário" do SIHSUS, foram identificados os prontuários dos pacientes selecionados nas unidades hospitalares, no intuito de proceder a uma averiguação da presença do diagnóstico de Aids, conforme os critérios vigentes de definição de caso, ${ }^{11,12}$ e avaliar a validade dos dados do SIH-SUS relacionados às variáveis demográficas e clínicas.

Para a obtenção dos dados nos prontuários, foi criado um instrumento de coleta com previsão de inclusão de informação referente a até três internações por paciente. Além das variáveis principais da AIH, foram contempladas as variáveis "endereço", "bairro", "escolaridade”, "ocupação", "estado civil”, “categoria de exposição", "teste anti-HIV" e, para cada internação, variáveis para registro de até quatro "diagnósticos secundários".

Os dados colhidos nos prontuários constituíram um novo banco de dados, tendo o paciente como unidade de observação. Esse banco foi utilizado nas análises desenvolvidas, prevendo para a variável dependente "status de notificação" a possibilidade de registro de três condições, conforme já mencionado: notificados, não notificados com evidência de diagnóstico de Aids e não notificados sem evidência para notificação.

\section{Análise dos dados}

Foi utilizado um modelo multinomial para a análise das variações nas chances de ocorrência de subnotificação de casos de Aids versus notificação e de não-notificação sem evidência de diagnóstico de Aids versus notificação, entre pacientes internados pelo SUS, no Município do Rio de Janeiro, em 1996, como função de variáveis demográficas, clínicas e organizacionais.

Os modelos multinomiais correspondem a generalizações dos modelos logísticos de resposta binária, permitindo a estimativa de respostas 
desordenadas e multicategóricas a partir do uso de variáveis explanatórias contínuas e categóricas. Para uma variável dependente com J categorias, estima-se J-1 equações logísticas diferentes, assumindo uma das categorias como base de referência. A cada uma das J-1 equações definidas, corresponde um vetor de parâmetros. ${ }^{1,2}$

No sentido de refletir o efeito do grau de utilização do hospital sobre a variável dependente, os pacientes que tiveram pelo menos duas internações convencionais no ano, em um mesmo hospital, foram diferenciados daqueles que tiveram somente uma internação convencional ou utilizaram apenas os serviços do hospital-dia.

As unidades hospitalares foram identificadas com base na consideração dos fluxos adotados para a notificação de casos de Aids. A presença de um setor de epidemiologia em funcionamento foi tratada como uma variável de interesse. Além disso, cada hospital foi classificado pela organização da atenção à Aids em uma das seguintes categorias: "apenas cadastrado" pelo Ministério da Saúde para emissão de AIH com procedimentos Aids; "com profissional de referência", quando possuía um ou mais especialistas treinados para o atendimento especializado de pacientes HIV/ Aids; e "com programa estabelecido", ou seja, capaz de desenvolver série de ações integradas e especializadas, de diagnóstico e de tratamento, que abrangem, além da internação, consultas ambulatoriais, hospitaldia ou assistência domiciliar.

Finalmente, houve preocupação em explicitar no modelo a qualidade da transcrição de dados do prontuário para a AIH (nome do paciente), como uma variável com potencial de explicação do status de notificação. Essa variável dicotômica foi identificada como "confere nome".

Os parâmetros do modelo proposto foram estimados a partir do método da máxima verossimilhança, usando o procedimento CATMOD do pacote estatístico SAS. ${ }^{14}$

\section{RESU LTAD O S}

O processo de revisão dos prontuários não somente possibilitou a reclassificação dos pacientes selecionados, como também a validação dos dados da AIH. Dos 165 pacientes não notificados e selecionados, 131 apresentaram, em seus prontuários, dados que possibilitaram contemplar os critérios de definição de caso de Aids. ${ }^{11,12}$ Os outros 34 pacientes foram classificados como não notificados sem evidências para definição de caso.
Em relação à confiabilidade do registro de procedimentos relacionados a Aids, foram identificados nove pacientes $(2,4 \%)$, cujas patologias motivadoras da internação não apresentavam nenhuma relação com HIV/ Aids. Esses pacientes foram internados em sete diferentes unidades hospitalares, sendo três universitárias, duas federais, uma estadual e uma municipal. Por outro lado, nos demais prontuários analisados, o registro dos procedimentos realizados e dos diagnósticos na AIH refletiu, precisamente, o conteúdo da documentação médica. A qualidade da informação nos prontuários era extremamente irregular, variando em organização, quantidade e conservação, até dentro do mesmo hospital. Quanto à conferência dos nomes dos pacientes, foi observado um índice de erro na transcrição de dados de $26,3 \%$, correspondendo a 99 pacientes, cuja grafia dos nomes na AIH diferia daquela registrada nos prontuários.

A Tabela 1 apresenta a distribuição de pacientes selecionados pelas variáveis consideradas na explicação da variação no nível de notificação da Aids. No que concerne às variáveis pertinentes aos indivíduos em si, verifica-se predominância do sexo masculino, numa proporção de 2:1; uma faixa etária de 81,4\% com idade entre 13 e 49 anos; predomínio de pacientes com ocupação não qualificada. Quanto à classificação das unidades hospitalares, verifica-se que a grande maioria dos pacientes foi internada em hospitais universitários ou municipais $(68,2 \%)$, e em hospitais com programa de Aids constituído (74,5\%).

Na Tabela 2 observa-se que a variável "sexo" não apresenta associação com a ocorrência de subnotificação, estando, no entanto, significativamente associada $(\mathrm{p}=0,0123)$ à ocorrência de não-notificação sem evidências para 'fechar caso' de Aids. As chances de subnotificação sem evidência de diagnóstico de Aids versus notificação entre mulheres são 2,9 vezes as mesmas entre homens.

No que concerne à variável "área de residência", foi encontrada uma associação negativa estatisticamente significante $(\mathrm{p}=0,0114)$ entre morar na área de planejamento sanitário (AP) 5-1, que corresponde à região administrativa (RA) de Bangu, e não estar notificado ao Sinan. As chances de subnotificação versus notificação são 71,3\% menores na AP5-1 do que no conjunto de AP que constitui o grupo de referência. Ser residente em outro município ou na AP4 (RA de Jacarepaguá e da Barra da Tijuca) está associado ao evento "não apresentar evidências para notificação", salientando-se que, no caso da AP4, as chances de ocorrência desse evento versus notificação são 10,7 vezes aquelas observadas entre os residentes nas $\mathrm{AP}$ de referência. 
Tabela 1 - Distribuição das variáveis contempladas no estudo da subnotificação de casos de Aids por unidades hospitalares relacionadas ao Sistema Único de Saúde, no Município do Rio de Janeiro, em 1996.

\begin{tabular}{|c|c|c|c|c|c|}
\hline Variável & $\mathrm{N}$ & $\%$ & Variável & $\mathrm{N}$ & $\%$ \\
\hline $\begin{array}{l}\text { Status de notificação } \\
\text { Não notificado } \\
\text { Sem evidências para notificação } \\
\text { N otificado }\end{array}$ & $\begin{array}{r}131 \\
34 \\
212\end{array}$ & $\begin{array}{r}34,8 \\
9,0 \\
56,2\end{array}$ & $\begin{array}{l}\text { Idade } \\
<13 \text { anos } \\
\quad 13-49 \text { anos } \\
>49 \text { anos }\end{array}$ & $\begin{array}{r}24 \\
307 \\
46\end{array}$ & $\begin{array}{r}6,4 \\
81,4 \\
12,2\end{array}$ \\
\hline $\begin{array}{l}\text { Sexo } \\
\text { Masculino } \\
\text { Feminino }\end{array}$ & $\begin{array}{l}251 \\
126\end{array}$ & $\begin{array}{l}66,6 \\
33,4\end{array}$ & $\begin{array}{l}\text { Resultado da internação } \\
\text { Alta/transferência } \\
\text { Ó bito }\end{array}$ & $\begin{array}{l}238 \\
139\end{array}$ & $\begin{array}{l}63,1 \\
36,9\end{array}$ \\
\hline $\begin{array}{l}\text { O cupação } \\
\text { Sem ocupação } \\
\text { Não qualificada } \\
\text { Semiqualificada } \\
\text { Qualificados manual } \\
\text { Qualificados não manual } \\
\text { Intermédio } \\
\text { Profissional } \\
\text { Ignorada }\end{array}$ & $\begin{array}{l}67 \\
84 \\
42 \\
41 \\
56 \\
11 \\
10 \\
66\end{array}$ & $\begin{array}{r}17,8 \\
22,3 \\
11,1 \\
10,9 \\
14,9 \\
2,9 \\
2,6 \\
17,5\end{array}$ & $\begin{array}{l}\text { Categoria de exposição } \\
\text { Homossexualismo masculino } \\
\text { Bissexualismo } \\
\text { Heterossexualismo } \\
\text { Uso de drogas endovenosas } \\
\text { Transfusão sangüínea } \\
\text { Transmissão vertical } \\
\text { Múltiplos fatores } \\
\text { Ignorado }\end{array}$ & $\begin{array}{r}51 \\
18 \\
115 \\
7 \\
8 \\
17 \\
8 \\
153\end{array}$ & $\begin{array}{r}13,5 \\
4,8 \\
30,5 \\
1,9 \\
2,1 \\
4,5 \\
2,1 \\
40,6\end{array}$ \\
\hline $\begin{array}{l}\text { Escolaridade } \\
\text { Analfabeto } \\
\text { Primeiro grau } \\
\text { Segundo grau } \\
\text { Superior } \\
\text { Ignorado }\end{array}$ & $\begin{array}{r}8 \\
79 \\
33 \\
15 \\
242\end{array}$ & $\begin{array}{r}2,1 \\
20,9 \\
8,8 \\
4,0 \\
64,2\end{array}$ & $\begin{array}{l}\text { Estado civil } \\
\text { Solteiro } \\
\text { Casado } \\
\text { Viúvo } \\
\text { Outro } \\
\text { Ignorado }\end{array}$ & $\begin{array}{r}218 \\
76 \\
18 \\
13 \\
52\end{array}$ & $\begin{array}{r}57,8 \\
20,2 \\
4,8 \\
3,4 \\
13,8\end{array}$ \\
\hline 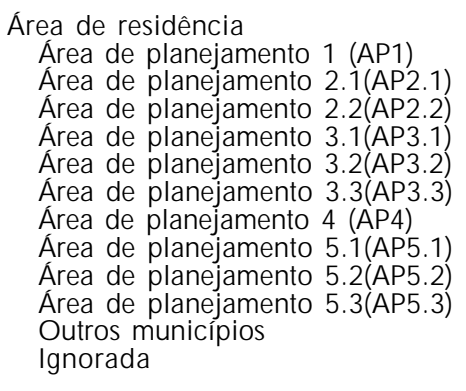 & $\begin{array}{r}38 \\
48 \\
14 \\
57 \\
27 \\
36 \\
25 \\
25 \\
12 \\
5 \\
86 \\
4\end{array}$ & $\begin{array}{r}10,1 \\
12,7 \\
3,7 \\
15,1 \\
7,2 \\
9,6 \\
6,6 \\
6,6 \\
3,2 \\
1,3 \\
22,8 \\
1,1\end{array}$ & $\begin{array}{l}\text { N atureza jurídica } \\
\text { Contratado } \\
\text { Estadual } \\
\text { Federal } \\
\text { Federal com verba própria } \\
\text { Universitário } \\
\text { Municipal } \\
\text { Utilização do hospital } \\
\text { Uma internação } \\
\text { Mais de uma internação } \\
\text { Hospital-dia } \\
\text { Oranizacão da atencão }\end{array}$ & $\begin{array}{r}11 \\
15 \\
17 \\
77 \\
142 \\
115\end{array}$ & $\begin{array}{r}2,9 \\
4,0 \\
4,5 \\
20,4 \\
37,7 \\
30,5\end{array}$ \\
\hline $\begin{array}{l}\text { Vigilância epidemiológica (VE) } \\
\text { Possui setor de VE } \\
\text { Não possui setor de VE }\end{array}$ & $\begin{array}{l}170 \\
207\end{array}$ & $\begin{array}{l}45,1 \\
54,9\end{array}$ & $\begin{array}{l}\text { O rganização da atenção } \\
\text { Apenas cadastrado } \\
\text { Profissional de referência } \\
\text { Programa constituído }\end{array}$ & $\begin{array}{r}63 \\
33 \\
281\end{array}$ & $\begin{array}{r}16,7 \\
8,8 \\
74,5\end{array}$ \\
\hline
\end{tabular}

Em relação à variável "categoria de exposição", ao se comparar a via de contágio heterossexual com todas as outras, observa-se a sua associação negativa $(\mathrm{p}=0,019)$ com as chances de ocorrência de não-notificação sem evidências para notificação versus notificação (razão de chances $=0,287$ ).

No que concerne à organização da atenção hospitalar aos pacientes HIV/Aids, foi encontrada uma associação significante em $\alpha=0,10$ entre a variável dependente e o fato do hospital apresentar um ou mais profissionais de referência para o tratamento da Aids. Apesar do modelo sugerir uma associação entre presença de programa de Aids na unidade hospitalar e menor subnotificação de casos, tal associação não possui significância estatística. Quando controlada pela variável "programa de Aids", a natureza jurídica municipal dos hospitais deixa de ser estatisticamente significante. No modelo multinomial, que não inclui a variável que reflete a organização da atenção hospitalar ao paciente HIV/ Aids, os pacientes internados em hospitais municipais apresentam chances $80 \%$ maiores $(\mathrm{p}=0,025)$, do que os pacientes internados no conjunto de hospitais estaduais, universitários, federais com verba própria e contratados que servem de referência, de não serem notificados ao Sinan versus serem notificados. Além disto, observa-se, no mesmo modelo, que entre os pacientes dos hospitais municipais há chances $100 \%$ maiores $(\mathrm{p}=0,087)$ de registro do evento 'não-notificação sem evidência para fechamento de caso'. No caso de inclusão e de exclusão da variável "organização da atenção", apontase para a associação estatisticamente significante ( $\mathrm{p}=0,051)$ entre a ocorrência de subnotificação e pacientes internados por Aids nos hospitais federais.

$\mathrm{Na}$ consideração da forma e do número de internações, cuja categoria de referência é hospital-dia, ou apenas uma internação no período estudado, foi encontrada uma associação bastante expressiva $(\mathrm{p}=0,003)$ entre ter sido internado mais de uma vez no mesmo hospital e estar notificado ao Sinan. Como esperado, foi encontrada associação negativa entre 
Tabela 2 - Modelo multinomial de estudo dos fatores associados à subnotificação de casos de Aids por unidades hospitalares relacionadas ao Sistema Ú nico de Saúde, no Município do Rio de Janeiro, 1996.

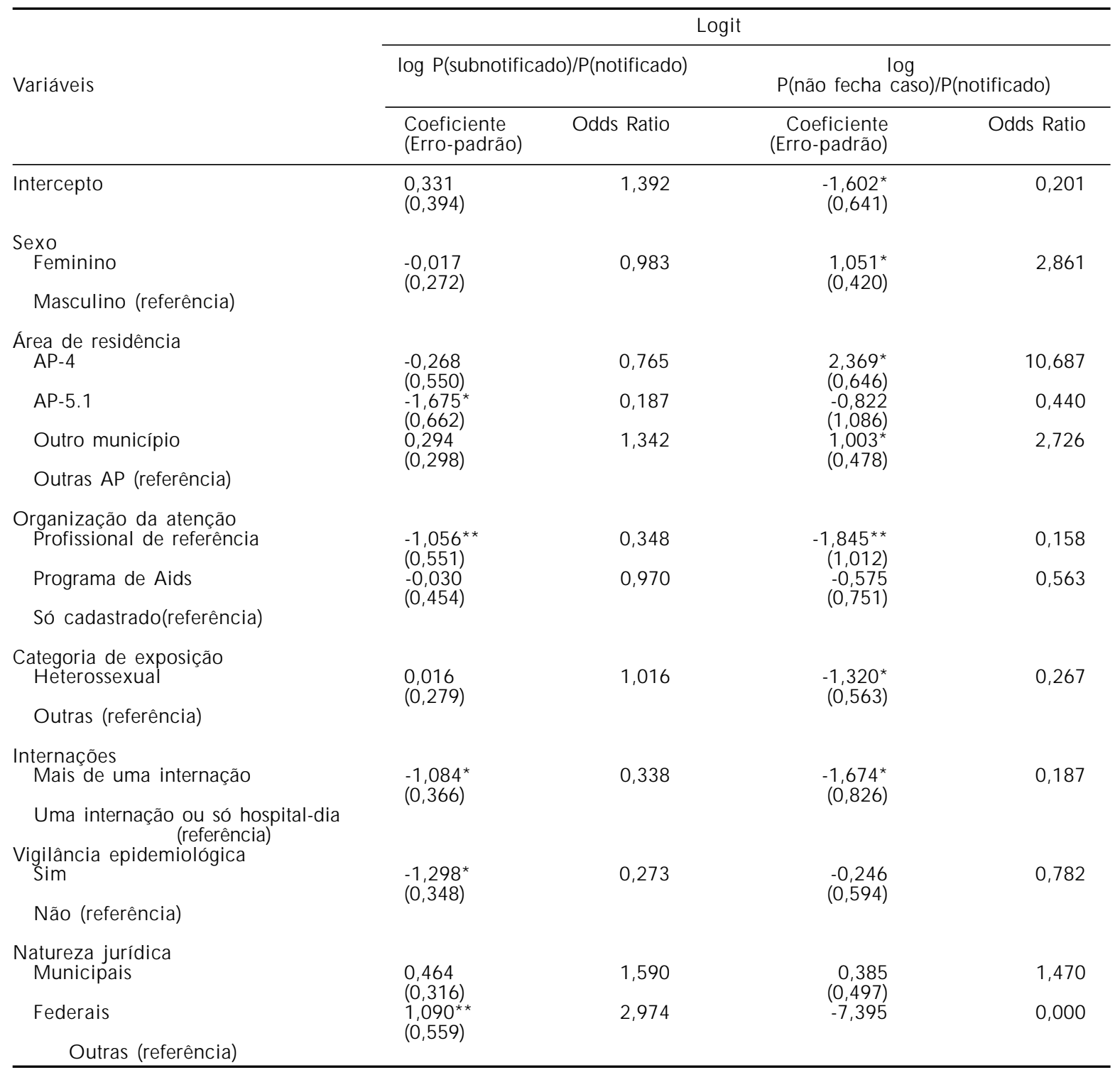

* Estatisticamente significante em $\alpha=0,05$

** Estatisticamente significante em $\alpha=0,10$

$A P$ - Área de Planejamento

sofrer mais de uma internação no ano e não apresentar evidências para 'fechar caso' de Aids (razão de chance $=0,187$ ).

Por fim, as chances de não ser notificado versus ser notificado, entre pacientes atendidos em hospitais com setor de vigilância epidemiológica em funcionamento, são 0,273 vezes as mesmas entre pacientes atendidos em hospitais sem um setor de vigilância epidemiológica $(\mathrm{p}=0,000)$.

Em outros modelos explorados não foram observadas quaisquer associações entre status de notificação e as variáveis idade, estado civil, escolaridade e ocupação.

\section{DISCU SSÃO}

Utilizando-se a definição de casos de Aids em vigor em 1996, menos sensível que a atual, foi encontrado um índice de $34,8 \%$ de subnotificação de pacientes que utilizaram a rede hospitalar vinculada ao SUS para tratamento da Aids, no Município do Rio de Janeiro. Esse fato, nada desprezível, aponta para um descompasso evidente entre a vigilância epidemiológica da Aids e a assistência aos pacientes HIV/ Aids, com repercussões na prevenção e no controle da epidemia.

Uma vez feito o diagnóstico de Aids, as características relacionadas ao indivíduo não influenciam o pro- 
cesso de notificação de pacientes com a doença. A subnotificação e o atraso no registro de casos de Aids estão significantemente associados aos aspectos estruturais e organizacionais dos hospitais, do próprio fluxograma do Sinan e da organização do SUS.

A baixa qualidade da informação médica e do registro dos dados nos sistemas de informação dificulta bastante a compatibilização dos bancos de dados. Levando-se em consideração que o prontuário médico é um documento que pertence ao paciente, e deve ser guardado pelo hospital por dez anos, explica o fato de não terem sido encontrados 13 prontuários da amostra.

Cabe considerar os mecanismos organizacionais informais e o trajeto percorrido pelas informações de saúde. O Ministério da Saúde recomenda um fluxo de formulários e de informações no Sinan, a partir da unidade de saúde. ${ }^{5}$ A Secretaria de Estado de Saúde do Rio de Janeiro, por meio de sua Assessoria de DST/Aids, acrescenta pequenas modificações e cada unidade hospitalar cria seus próprios atalhos. Em alguns casos, apesar da ficha de notificação ter sido preenchida por ocasião da internação, o paciente ainda não constava do banco de dados do Sinan municipal e a ficha ainda seguia seu fluxo interno ou externo ao hospital. Em outros casos, as fichas são entregues diretamente ao gerente do programa municipal de Aids pelo setor de vigilância epidemiológica. Além disso, observou-se que o turn-over de profissionais no programa municipal ou nos setores de vigilância epidemiológica altera o fluxo da informação e, conseqüentemente, o tempo decorrido entre o preenchimento da ficha de notificação e a entrada de dados no Sinan.

Os achados referentes à área de residência dos pacientes necessitariam ser melhor explorados. Uma hipótese seria a de que os pacientes residentes na AP4 tivessem acesso mais precocemente aos cuidados hospitalares, devido a uma melhor organização da rede hospitalar naquela região, o que justificaria maiores chances dos pacientes serem internados sem apresentar um diagnóstico definitivo de Aids.

A consideração das questões gênero-específicas na Aids foi sendo introduzida a reboque das mudanças de

\section{REFERÊN CIAS}

1. Agresti A. Categorical data analysis. New York: John Wiley and Sons; 1990.

2. Aldrich JH, Nelson FD. Linear probability, logit and probit models. Newbury Park: Sage Publications; 1984. perfil da epidemia. A classificação das categorias de exposição e, principalmente, as definições de caso não contemplavam as especificidades das pacientes de sexo feminino. ${ }^{15}$ Isso poderia explicar a associação positiva da variável "sexo feminino" com o evento "sem evidência para notificação", já que, com a definição de caso de Aids utilizada em 1996, ficavam excluídas patologias associadas à imunodeficiência em mulheres, como o carcinoma invasivo de colo de útero.

O presente trabalho foi desenvolvido com base nos dados do SIH-SUS para o período de 1996. Dada a restrição das informações daquelas unidades hospitalares cadastradas pelo Ministério da Saúde para emissão de AIHs, com procedimentos relacionados ao tratamento da Aids, acredita-se que exista percentual de pacientes com Aids internados em outros hospitais que não puderam ser analisados. Trata-se, ainda, de um estudo limitado ao Município do Rio de Janeiro e seus resultados precisariam, portanto, ser confrontados com outros estudos regionais, a fim de se obter uma expressividade maior.

O índice de subnotificação de casos de Aids descrito no presente estudo, somado ao índice de 51,9\% de subnotificação encontrado por Lemos $^{9}$ ao comparar as informações do Sistema de Informação sobre Mortalidade (SIM), com os dados do Sinan para o Estado do Rio de Janeiro, no período de 1991-1995, aponta para a necessidade de se criarem mecanismos que melhorem a qualidade do sistema de notificação, agilizando a coleta e a disponibilização das informações em saúde. Visto que os aspectos organizacionais apresentam importante associação com a subnotificação de casos de Aids, seria apropriado uniformizar um fluxograma de informações para o Sinan, no Estado do Rio de Janeiro, e investir na manutenção de setores de vigilância epidemiológica em todos os hospitais cadastrados para atender pacientes com HIV/Aids, a fim de reduzir o atraso de registro e a subnotificação de casos de Aids no Rio de Janeiro. Os treinamentos fornecidos pelas Secretarias Municipal e Estadual de Saúde aos médicos que atendem pacientes com HIV/Aids deveriam incluir, obrigatoriamente, conteúdo relativo à vigilância epidemiológica e ao Sinan. Os recursos envolvidos no controle e na assistência a Aids são de elevada monta e precisam ser racionalmente alocados, a fim de se tornarem eficazes e alcançarem o impacto desejado.
3. Barbosa MTS, Struchiner CJ. Estimativas do número de casos de Aids: comparação de métodos que corrigem 0 atraso da notificação. In: Simpósio Satélite. A Epidemia da Aids no Brasil: Situação e Tendências. Brasília: Ministério da Saúde; 1997. p. 15-26. 
4. Bastos C, Galvão J, Pedrosa JS, Parker R. Introdução. In: Parker R, Bastos C, Galvão J, Pedrosa JS. A Aids no Brasil (1982-1992). Rio de Janeiro: ABIA/IMS/UERJ/ Relume-Dumará; 1994. p.13-56.

5. Carvalho DM. Sistemas de informação e alocação de recursos: um estudo sobre as possibilidades de uso das grandes bases de dados nacionais para uma alocação orientada de recursos [dissertação]. Rio de Janeiro: Universidade do Estado do Rio de Janeiro - Instituto de Medicina Social; 1998.

6. Cochran WG. Sampling techniques. New York: John Wiley; 1977.

7. Dourado MIC Barreto ML, Almeida-Filho N, Biehl JG, Cunha S. Região Nordeste. In: Simpósio Satélite. A Epidemia da Aids no Brasil: Situação e Tendências. Brasília: Ministério da Saúde; 1997. p.123-44.

8. Ferreira VMB, Portela MC. Avaliação da subnotificação de casos de Aids no Município do Rio de Janeiro com base em dados do Sistema de Informações Hospitalares do Sistema Ú nico de Saúde. Cad Saúde Pública 1999;15:317-24.

9. Lemos KRV. Mortalidade por Aids no Estado do Rio de Janeiro e avaliação do sub-registro de casos de Aids tendo como indicador a declaração de óbito [dissertação]. Rio de Janeiro (RJ): U niversidade do Estado do Rio de Janeiro - Instituto de Medicina Social; 1998.
10. Levcovitz E, Pereira TRC. SIH -SU S (Sistema AIH): uma análise do sistema público de remuneração de internações hospitalares no Brasil - 1983/1991. Rio de Janeiro: Instituto de Medicina Social da UERJ; 1993. (Estudos em Saúde Coletiva, 57).

11. Ministério da Saúde. Revisão da definição nacional do caso de Aids em adultos. Brasília; 1992.

12. Ministério da Saúde. Revisão da definição nacional do caso de Aids em crianças. Brasília; 1994.

13. Proietti FA, Caiaffa WT. Minas Gerais. In: Simpósio Satélite. A Epidemia da Aids no Brasil: Situação e Tendências. Brasília: Ministério da Saúde; 1997. p. 193-216.

14. SAS Language, SAS/STAT user's guide, version $6.4^{\text {th }}$ ed. Cary: SAS Institute; 1990.

15. Wortley PM, Chu SY, Berkelman RL. Epidemiology of HIV/AIDS in women and the impact of the expanded 1993 CDC surveillance definition of AIDS. In: Cotton $D$, Watts DH. The medical management of AIDS in women. New York: Wiley-Liss; 1997. p. 3-14. 